\title{
Star Formation and the Atomic-Molecular Transition
}

\author{
Mark R. Krumholz \\ Department of Astronomy, University of California, Santa Cruz, \\ Santa Cruz, CA 95064, USA \\ email: krumholz@ucolick.org
}

\begin{abstract}
In the last decade large surveys have allowed us to separate the atomic and molecular phases of the interstellar media in a wide variety of galaxies, and to determine how each of these phases correlates with star formation. The most striking results of these observations have been that the transition from $\mathrm{H}$ I to $\mathrm{H}_{2}$ occurs at a characteristic gas column density that depends on metallicity, and that star formation correlates primarily with the molecular phase. These observations have stimulated a burst of theoretical work, which I review here. The work can be broken into three primary questions: what physical mechanisms control the $\mathrm{H}$ i to $\mathrm{H}_{2}$ transition? Why does star formation correlate with $\mathrm{H}_{2}$ and not with some other phase of the ISM? Finally, what are the implications of the answers to the previous two questions for our understanding of star formation on the cosmological scale? I discuss our current best answers to each of these questions, and conclude with prospects for future work.
\end{abstract}

Keywords. galaxies: formation — ISM: atoms — ISM: molecules — stars: formation

\section{Introduction}

Ever since the advent of spatially resolved maps of star formation, atomic gas, and molecular gas in nearby galaxies, it has been clear that star formation is closely associated with the molecular phase of the interstellar medium, and only poorly correlated with the atomic phase. This result was demonstrated using azimuthally-averaged measurements by Wong \& Blitz (2002), and later by the point-by-point measurements of Kennicutt et al. (2007), Bigiel et al. (2008), and Leroy et al. (2008). This remains true even in the outer, H I-dominated parts of spiral galaxies, where star formation does start to correlate with $\mathrm{H}$ I, but apparently only because $\mathrm{H}$ I itself becomes correlated with $\mathrm{H}_{2}$ (Bigiel et al. 2010; Schruba et al. 2011). These measurements were all made using CO emission as a proxy for $\mathrm{H}_{2}$, leaving unanswered the question of whether the true correlation is between star formation and $\mathrm{H}_{2}$, or star formation and $\mathrm{CO}$. However, subsequent measurements using other proxies to disentangle $\mathrm{CO}$ from $\mathrm{H}_{2}$ in low metallicity galaxies where the two are not co-extensive have demonstrated that the true correlation is with $\mathrm{H}_{2}$ (Bolatto et al. 2011; Krumholz et al. 2011; Leroy et al. 2011).

Based on these local observations, a first-order description of star formation in Local Group galaxies is simply that the star formation rate per unit area is $\dot{\Sigma}_{*}=f_{\mathrm{H}_{2}} \Sigma_{\text {gas }} / 2$ Gyr, where $\Sigma_{\text {gas }}$ is the total gas surface density, and $f_{\mathrm{H}_{2}}$ is the fraction of that gas in the form of $\mathrm{H}_{2}$. This model is clearly not the full story of star formation - the star formation timescale is not fixed to 2 Gyr when one examines a wider sample of galaxies (e.g. Saintonge et al. 2011), and even in the Local Group there are interesting deviations from this result (see the contribution by S. Meidt in this volume). Nonetheless, that such a first-order description is even roughly accurate implies that the atomic to molecular transition in galaxies must be somehow deeply related to the onset of star formation.

This observational result poses a three-fold challenge for theory, and in this review I discuss the status of attempts to meet this challenge. First, in $\S 2$ I address the question 
of what physical processes set $f_{\mathrm{H}_{2}}$, and whether we can develop a model to predict its value from other properties of a galaxy. Second, $\S 3$ asks why does star formation care about $\mathrm{H}_{2}$ at all? The answer to this question is not at all obvious, and turns out to be quite subtle. Third, in $\S 4$ I discuss the implications of these results for star formation on cosmological scales. Finally, in $\S 5$ I discuss prospects for future work.

\section{What Determines the Molecular Fraction?}

The chemical state of interstellar hydrogen is governed by the balance between two processes: dissociation of $\mathrm{H}_{2}$ molecules by photons in the Lyman-Werner bands around $1000 \AA$, and formation of $\mathrm{H}_{2}$ molecules, which in galaxies with a metallicity above $\sim 10^{-5}$ of Solar occurs predominantly on the surfaces of dust grains (Omukai et al. 2010). For the ultraviolet radiation field observed in the Solar neighborhood, the dissociation rate is roughly $5 \times 10^{-11} \mathrm{~s}^{-1}$ per $\mathrm{H}_{2}$ molecule (Draine \& Bertoldi 1996), while the $\mathrm{H}_{2}$ formation rate per $\mathrm{H}$ atom is roughly $3 \times 10^{-15} Z^{\prime} n_{2} \mathrm{~s}^{-1}$, where $n_{2}$ is the $\mathrm{H}$ I number density in units of $100 \mathrm{~cm}^{-3}$ and $Z^{\prime}$ is the metallicity relative to Solar (Wolfire et al. 2008). (Note that we are implicitly assuming that dust abundance is proportional to metallicity.) Balancing these two rates suggests that the $\mathrm{H}_{2}$ fraction at Solar metallicity should be extremely low except at extremely high densities: $f_{\mathrm{H}_{2}} \sim 10^{-4} n_{2}^{-1}$.

However, Lyman-Werner band photons are very sensitive to extinction by dust, which provides a cross-section $\sigma_{d} \approx 10^{-21} \mathrm{~cm}^{2}$ per $\mathrm{H}$ atom. In regions of high column density, the UV radiation field can be attenuated enough for $f_{\mathrm{H}_{2}}$ to approach unity, leading to formation of a molecular cloud. Such clouds therefore tend to have a characteristic structure of an outer layer where the interstellar UV field is weakly attenuated and the hydrogen is primarily $\mathrm{H} \mathrm{I}$, and a highly-shielded inner region where $\mathrm{H}_{2}$ predominates. A large number of authors have studied the structure of such clouds, using levels of precision from analytic approximations to detailed quantum mechanical treatments of the radiation and level populations, and geometries from simple slabs to spheres to turbulent media (e.g. van Dishoeck \& Black 1986; Sternberg 1988; Draine \& Bertoldi 1996; Neufeld \& Spaans 1996; Liszt 2002; Glover \& Mac Low 2007; Krumholz et al. 2008; Gnedin et al. 2009; McKee \& Krumholz 2010; Mac Low \& Glover 2012, to name but a small sample).

In general the results depend on the gas volume and column densities and the ambient radiation field, but Krumholz et al. (2009b) simplified the problem considerably by pointing out that to first order only the ratio of volume density to radiation field strength matters, and that, for cold atomic hydrogen in two-phase equilibrium with warm H I, this ratio is nearly constant. This allows one to define a characteristic column density $\Sigma_{\mathrm{HI}-\mathrm{H}_{2}} \sim 10 / Z^{\prime} M_{\odot} \mathrm{pc}^{-2}$ of $\mathrm{H}$ I required to shield an $\mathrm{H}_{2}$ cloud. (See McKee \& Krumholz (2010) for a more precise formula.) The metallicity enters the problem through the dependence of both the $\mathrm{H}_{2}$ formation rate and the dust shielding on the abundance of dust grains, which in the model is presumed to scale with the metallicity.

As illustrated in Figure 1, this model has successfully predicted the chemical state of the interstellar medium in the Milky Way (Lee et al. 2012), nearby galaxies (Fumagalli et al. 2010; Bolatto et al. 2011), and the high-redshift universe (Krumholz et al. 2009a). In particular, the predicted increase in the $\mathrm{H}$ I column required for $\mathrm{H}_{2}$ to form at subSolar metallicity has been confirmed by observation. Models that incorporate the $\mathrm{H}$ I to $\mathrm{H}_{2}$ into their recipe for star formation have also been successful in explaining, and in some cases predicting, observed changes in the star formation law as a function of galaxy metallicity (e.g. Robertson \& Bullock 2008; Gnedin et al. 2009; Krumholz et al. 2009c).

A final caution is in order on these results. The analytic models shown in Figure 1 assume that the gas is in chemical equilibrium, but this need not always be the case. 

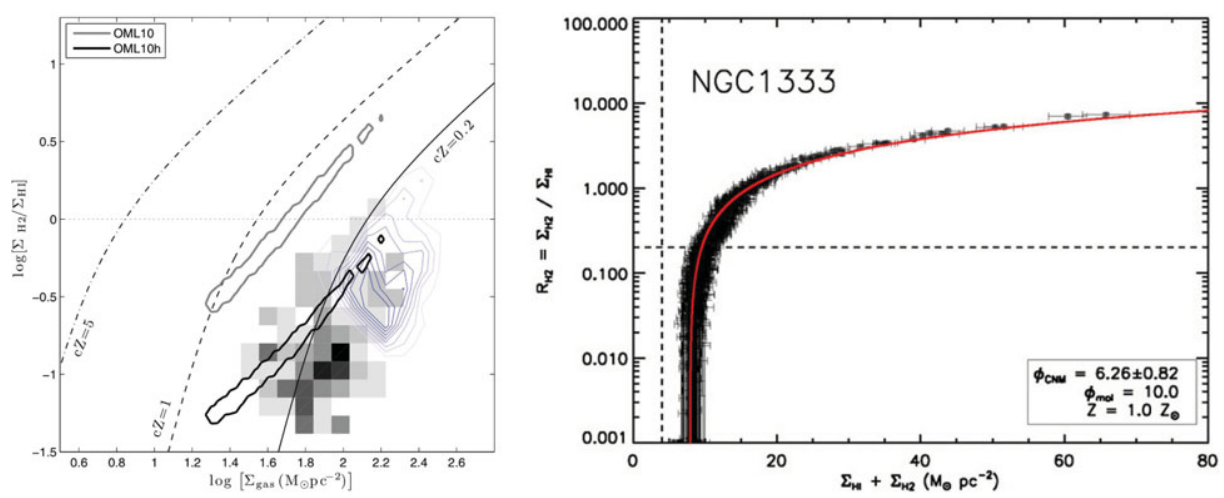

Figure 1. Observations of the $\mathrm{H}$ I to $\mathrm{H}_{2}$ transition. Both panels show the ratio of $\mathrm{H}_{2}$ to $\mathrm{H}$ I column density $R_{\mathrm{H}_{2}}=\Sigma_{\mathrm{H}_{2}} / \Sigma_{\mathrm{HI}}$ versus the total gas column density $\Sigma_{\mathrm{H}_{2}}+\Sigma_{\mathrm{HI}}$. The left panel, taken from Bolatto et al. (2011), shows observations of the Small Magellanic Cloud averaged on $12 \mathrm{pc}$ and $200 \mathrm{pc}$ scales (blue contours and grayscale boxes, respectively). The line labelled $c Z=0.2$ is the prediction of the KMT model (Krumholz et al. 2008, 2009b; McKee \& Krumholz 2010) at SMC metallicity, and the dashed lines are predictions at Solar and $5 \times$ Solar metallicity. The right panel, taken from Lee et al. (2012), shows observations of sightlines toward the cloud NGC 1333 (black points with error bars), compared to the KMT model prediction (red line). Note the factor of $\sim 5$ shift between the two panels in the value of $\Sigma_{\mathrm{H}_{2}}+\Sigma_{\mathrm{HI}}$ for which $R_{\mathrm{H}_{2}}=1$, which is the result of the SMC's metallicity of $\sim 0.2 \times$ Solar. Both panels are reproduced by permission of the AAS.

Formation and dissociation of $\mathrm{H}_{2}$ is a rather slow process. The rate given above for $\mathrm{H}_{2}$ formation implies that the time required to convert a fully atomic region to fully molecular, even in the absence of dissociation, is $\sim 10 Z^{\prime-1} n_{2}^{-1} \mathrm{Myr}$, which approaches typical molecular cloud lifetimes of 30 Myr (Kawamura et al. 2009) even at Solar metallicity. Molecule formation, like any reaction whose rate scales as the square of density, can be accelerated by turbulent compression, but nonetheless it remains a difficult question whether the typical region of the ISM can reach chemical equilibrium.

Numerical simulations by Krumholz \& Gnedin (2011) suggest that, averaged over $\sim 100 \mathrm{pc}$ scales, the ISM does reach chemical equilibrium except at metallicities below about $1 \%$ of Solar. Mac Low \& Glover (2012) reach the opposite conclusion from their simulations on scales below $\sim 10 \mathrm{pc}$, and argue that most star-forming clouds do not reach chemical equilibrium before they are disrupted by stellar feedback; in this picture $f_{\mathrm{H}_{2}}$ is set by the lifetimes of star-forming clouds, which dictates how much of the gas is able to go molecular before the cloud is disrupted. However, this result seems extremely difficult to reconcile with the observational results shown in Figure 1, which indicate that on scales of $10 \mathrm{pc}$ (for the SMC) and $<1 \mathrm{pc}$ (for NGC 1333) the equilibrium models are very accurate. This success of the simpler equilibrium models in matching observations on small scales, and the failure of the time-dependent ones, remains an unsolved problem. However, both models and observation are in agreement that the equilibrium models are reasonable over 100 pc scales or larger.

\section{Why Does $\mathbf{H}_{2}$ Correlate with Star Formation?}

The preceding question answers, at least in part, the question of where the ISM will be $\mathrm{H}_{2}$ and where it will be $\mathrm{H}$, but we have not yet addressed the question of why the chemical state matters at all. On its face, this is surprising. The atomic to molecular transition changes the gas sound speed by a factor of 1.3 (due to the change in mean 

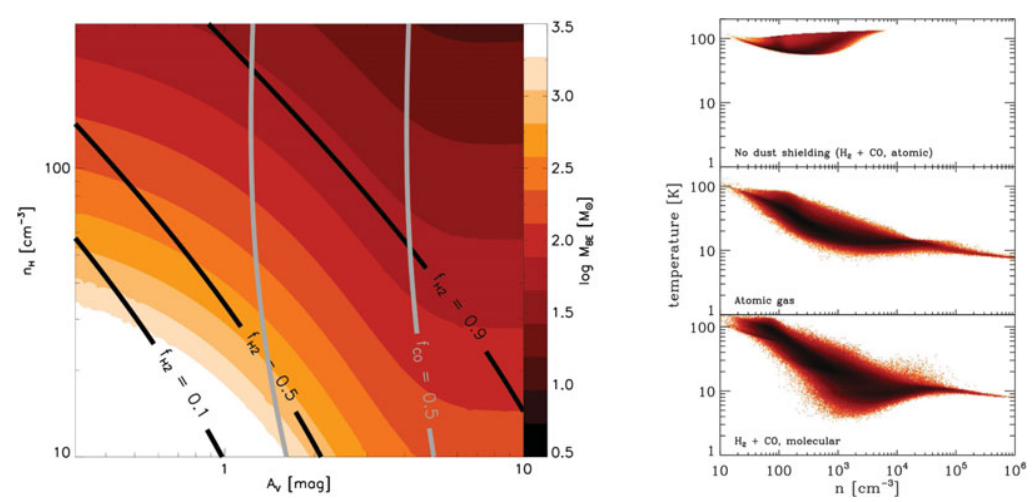

Figure 2. Left: contours show the Bonnor-Ebert mass at the center of a cloud with visual extinction $A_{V}$ and number density of $\mathrm{H}$ nuclei $n_{\mathrm{H}}$ as indicated on the $x$ and $y$ axes. This is the maximum mass than can be supported against collapse by thermal pressure. Black contours labeled $f_{\mathrm{H}_{2}}=0.1,0.5$, and 0.9 show the molecular gas fraction in the cloud. Note that generally clouds with $f_{\mathrm{H}_{2}}<0.1$ have $M_{\mathrm{BE}}>10^{3} M_{\odot}$, while those $f_{\mathrm{H}_{2}}>0.9$ have $M_{\mathrm{BE}}<50 M_{\odot}$, indicating a dramatic loss of pressure support. Figure adapted from Krumholz et al. (2011) and reproduced by permission of the AAS. Right: temperature-density distributions from three simulations of a turbulent medium with different chemical and thermal processes. The bottom panel shows a simulation including $\mathrm{H}_{2}$ and $\mathrm{CO}$ formation and cooling, and radiative heating attenuated by dust. The middle panel shows a simulation without $\mathrm{H}_{2}$ or $\mathrm{CO}$ included, but with atomic cooling and dust attenuation. The top panel shows a simulation with no dust attenuation. Note that in the top panel there is no dense, cold gas, while in the bottom two there is a tail of gas at high density and low temperature that is collapsing to form stars. Figure adapted from Glover \& Clark (2012a), and reproduced by permission.

molecular weight), but this is clearly not a major difference. Since $\mathrm{H}_{2}$ is not a particularly good coolant at low temperatures, there is no large corresponding change in temperature. Why then does star formation follow $\mathrm{H}_{2}$ so closely, and not $\mathrm{CO}$ (which is a good coolant) or $\mathrm{H} \mathrm{I}$ (which is the bulk of the mass in most galaxies)?

The answer to this question has been supplied by two recent papers (Krumholz et al. 2011; Glover \& Clark 2012a). The key turns out to be the role of dust shielding in controlling both the chemical and the thermal state of the ISM. The chemical state, as discussed above, turns on the balance between photodissociation and $\mathrm{H}_{2}$ formation on dust grains. The thermal state of the neutral ISM involves a similar balance between heating by the grain photoelectric effect and cooling by collisionally-excited line emission. The dependences of the chemical and thermal processes on the volume density, column density, and radiation field are strikingly similar. Line cooling and $\mathrm{H}_{2}$ formation are both collisional processes whose rates depend on density and metallicity as $n^{2} Z^{\prime}$ (again assuming that the grain-to-metals ratio is constant). Photodissociation and grain photoelectric heating are both dominated by photons with wavelengths near $1000 \AA$, and thus depend on the radiation field and the amount of dust attenuation in similar ways.

Because of these similarities, gas temperatures and chemical states are very wellcorrelated over a very broad range of gas densities, dust extinctions, and metallicities. A transition from $\mathrm{H}$ I to $\mathrm{H}_{2}$ is almost always accompanied by a change in temperature from $>100 \mathrm{~K}$ to $\sim 10 \mathrm{~K}$. The underlying driver of this behavior is primarily dust shielding: if a region is well-shielded enough for $\mathrm{H}_{2}$ molecules to be the dominant state of hydrogen, then it is also well-shielded enough to allow very low temperatures. The converse is true as well: poorly shielded regions receive heating rates high enough to render them warm. Figure 2 illustrates this behavior. This explains the $\mathrm{H}_{2}$-star formation correlation: the 
transition from $\mathrm{H}$ I to $\mathrm{H}_{2}$ does not cause the onset of star formation, but the drop in gas temperature that accompanies the transition does.

The idea that the underlying variable controlling star formation is gas temperature and not chemical state yields an interesting prediction (Glover \& Clark 2012b; Krumholz 2012). As discussed above, the timescale for gas to become molecular is relatively long, and can be comparable to the dynamical times of star-forming clouds, particularly at low metallicity. In contrast, the timescale for gas to reach thermal equilibrium is a factor of $\sim 10^{3}$ shorter. In the Milky Way this difference in timescales does matter, because both the chemical and thermal timescales are smaller than the timescale over which clouds form stars. However, the chemical and thermal timescales are both proportional to the metallicity, while the dynamical time (and thus presumably the star formation time) is not. There must be metallicities at which the dynamical time is intermediate between the thermal and chemical timescales. If temperature and not chemical state is what controls star formation, at these metallicities we expect that star formation will proceed before the gas is able to turn molecular, and should therefore correlate with the cold atomic phase of the ISM, not the molecular phase. Simulations by Glover \& Clark (2012b) bear out this suggestion, and the analytic models of Krumholz (2012) indicate that this effect should begin to appear at metallicities between $1 \%$ and $10 \%$ of Solar.

\section{What are the Implications of These Results for Star Formation over Cosmic Time?}

The final question for theory to address is the significance of the atomic to molecular transition for star formation on the large scale. Observations of the SMC (Bolatto et al. 2011) provide unambiguous evidence that low-metallicity dwarf galaxies such as the SMC do not follow the Kennicutt (1998) star formation relation to which, until the last few years, almost all numerical simulations and semi-analytic models of galaxy evolution were calibrated. We have a theoretical model that can explain the SMC data, and that predicts even more extreme deviations from the Kennicutt relation in lower metallicity systems, which were more common at high redshift. If we model star formation over cosmic time using this improved model, what changes?

One clear answer seems to be that the star formation law in high-redshift systems is modified. Observations of damped Lyman $\alpha$ systems at $z \sim 2-3$ show far less star formation at a given column of $\mathrm{H}$ I than would be a naive extrapolation of the total gasKennicutt (1998) relation would predict (Wolfe \& Chen 2006; Wild et al. 2007; Rafelski et al. 2011). Both simulations (Gnedin et al. 2009; Gnedin \& Kravtsov 2010; Kuhlen et al. 2012) and analytic models (Krumholz et al. 2009a) have demonstrated that this results from the systematically lower metallicities of DLAs compared to modern-day galaxies. Low metallicities result in a lower fraction of cold, star-forming $\mathrm{H}_{2}$ at a given $\mathrm{H}$ I column.

A metallicity-dependent star formation law may also help explain a number of other observations. For example, the observed star formation rate density in the Universe peaks at $z \sim 1-3$ and falls off at higher $z$ (e.g. Hopkins \& Beacom 2006; Bouwens et al. 2010). However, the accretion rate of gas onto dark matter halos should monotonically increase with $z$ (e.g. Neistein et al. 2006), so something must be preventing gas that falls onto dark matter halos from forming stars efficiently above $z \sim 3$. Similarly, the observed galaxy mass function has a faint-end slope considerably shallower than is expected for the mass function of small dark matter halos. As a result both semi-analytic models (e.g. Cirasuolo et al. 2010) and simulations (e.g. Choi \& Nagamine 2012) tend to significantly overproduce dwarf galaxies, even with careful fine-tuning of the feedback parameters. Several authors have pointed out that these and a number of other observations can be 

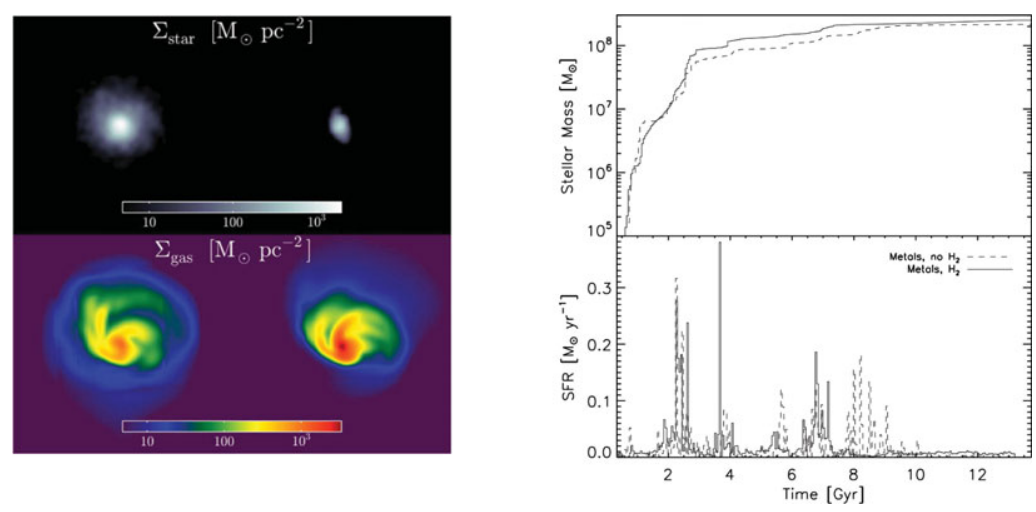

Figure 3. Left: A comparison of two simulations of a dwarf galaxy at $z=4$, from Kuhlen et al. (2012). The left column shows the surface density of stars (top) and gas (bottom) in a simulation using a conventional star formation recipe that does not depend on metallicity, while the right column shows the same galaxy in an otherwise-identical simulation where the star formation law is metallicity-dependent. Reproduced by permission of the AAS. Right: a comparison of the stellar mass (top) and star formation rate (bottom) versus cosmic time in two simulated dwarf galaxies from Christensen et al. (2012). The simulations are identical except that the one illustrated by the solid line uses a metallicity-dependent star formation law, while the other uses a metallicity-independent star formation law. Note that, in comparison to the simulations shown on the left where changing from a metallicity-independent to a metallicity-dependent star formation recipe clearly affects the stellar content of the galaxy, for the Christensen et al. simulations there is almost no effect. Reproduced by permission.

explained if the efficiency with which dark matter halos turn gas into stars is a strong function of halo mass, with the efficiency peaking at $\sim 10^{10-11} M_{\odot}$ (Bouché et al. 2010; Behroozi et al. 2012). Krumholz \& Dekel (2012) argue that metallicity-dependent star formation provides a natural physical mechanism for this, since small halos have trouble holding onto their metals, and thus tend to be lower metallicity.

Numerical simulations are somewhat divided on whether this metallicity-quenching hypothesis is correct. Kuhlen et al. (2012, figure 3, left panel) perform high-resolution simulations of high- $z$ dwarf galaxies in a small cosmological volume, and find that a metallicity-dependent star-formation recipe does indeed suppress the formation of dwarf galaxies and the overall star formation rate at high $z$, yielding better (though by no means perfect) agreement with observations than a model with a metallicity-independent star formation recipe. On the other hand, Schaye et al. (2010), in their lower-resolution simulations of larger cosmological volumes, find that a metallicity-dependent star formation recipe makes almost no difference to their cosmic star formation histories. Similarly, Christensen et al. (2012, figure 3, right panel) conduct high resolution zoom-in simulations of a single dwarf galaxy down to $z=0$. They find that the total stellar mass buildup of the galaxy is not significantly modified by a metallicity-dependent star formation recipe, although the morphology of the galaxy is.

The most likely explanation for the discrepant results is the difference in star formation feedback recipes in the simulations. Both Schaye et al. and Christensen et al. rely on supernova feedback recipes that are very efficient, and include a variety of tricks (e.g. temporarily turning off radiative cooling in supernova-heated gas) to enhance the effectiveness of supernova blast waves that are inadequately resolved in the simulations. When these recipes are in place, self-regulation of star formation is so strong that the small-scale star formation law does not matter much. A less efficient star formation law 
results in less matter ejection by strong winds, and this pushes the star formation rate back up; a more efficient star formation recipe produces more winds, which lowers the star formation rate. In contrast, Kuhlen et al. (2012) avoids these tricks in order to allow a cleaner experiment on the effects of metallicity-dependent star formation. As a result, supernova feedback is far less effective at regulating star formation, and changing the star formation law to make it metallicity-dependent does matter.

Which simulations are closer to reality is unclear. Supernova feedback in the Kuhlen et al. simulations is clearly too weak due to resolution problems, but the recipes in Schaye et al. and Christensen et al. could easily be too strong. They are dependent on a number of free parameters that have been calibrated so that simulations using a Kennicutt (1998)based recipe for star formation will match observations as closely as possible. However, this means that the level of feedback has been turned up very high in part to compensate for the (incorrect) assumption that stars form in $\mathrm{H}$ I as well as they do in $\mathrm{H}_{2}$. Until the calibration exercise is repeated with a star formation model that correctly includes the distinction between warm, non-star-forming $\mathrm{H}$ I and cold, star-forming $\mathrm{H}_{2}$, we will not know if the free parameters in the supernova feedback recipes are reasonable.

\section{Future Prospects}

Given the preceding discussion, what is the way forward? One clear need is to extend our observations to lower metallicities in order to provide more stringent constraints on the theoretical models. While the models have been very successful at Solar metallicity, the SMC remains the sole comprehensive test at substantially sub-Solar metallicity. Other low-metallicity tests thus far are limited to rough consistency checks (e.g. Fumagalli et al. 2010), simply because the data quality is so much lower than what is available for the SMC. Hopefully this will change in the era of ALMA and Herschel, and it will become possible to replicate Bolatto et al. (2011)'s analysis of the SMC at even lower metallicities.

For simulations targeting sub-galactic scales, the inconsistency of the time-dependent models (Mac Low \& Glover 2012) and the observations (Bolatto et al. 2011; Lee et al. 2012) stands out as the single biggest challenge. The equilibrium models seem to work even down to sub-pc scales, which suggests that either we are misinterpreting the observations, or that the simulations are missing something fundamental. On the side of the former is the possibility that we are underestimating the $\mathrm{H}$ I column due to self-absorption (Braun 2012), though it would seem to require a conspiracy for this effect to trick galactic and extragalactic observations at two different resolutions and metallicities into matching the models as well as they do. Another possibility is that the time-dependent simulations are assuming molecular cloud lifetimes that are too short, and/or that their treatment of the radiative transfer of dissociating photons is too simplistic.

For cosmological simulations and models, the most urgent problem is to resolve the disagreement about how much a metallicity-dependent star formation law matters. Clearly it is required to explain the DLA observations, but this does not necessarily imply that it makes a difference for quantities like the galaxy mass function or the cosmic star formation history. Resolving this will require study of the interactions between changes in the star formation law and changes in feedback recipes. The challenge will be to match both the observed metallicity-dependent star formation laws in individual galaxies and galaxy statistics measured on cosmological scales.

\section{Acknowledgements}

This work was supported by the Alfred P. Sloan Foundation, the NSF through grant CAREER-0955300, and NASA through ATFP grant NNX09AK31G. 


\section{References}

Behroozi, P. S., Wechsler, R. H., \& Conroy, C. 2012, ApJL, submitted, arXiv:1209.3013

Bigiel, F., Leroy, A., Walter, F., et al. 2010, AJ, 140, 1194

-. 2008, AJ, 136, 2846

Bolatto, A. D., Leroy, A. K., Jameson, K., et al. 2011, ApJ, 741, 12

Bouché, N., Dekel, A., Genzel, R., et al. 2010, ApJ, 718, 1001

Bouwens, R. J., Illingworth, G. D., Oesch, P. A., et al. 2010, ApJL, 709, L133

Braun, R. 2012, ApJ, 749, 87

Choi, J.-H. \& Nagamine, K. 2012, MNRAS, 419, 1280

Christensen, C., Quinn, T., Governato, F., et al. 2012, MNRAS, 425, 3058

Cirasuolo, M., McLure, R. J., Dunlop, J. S., et al. 2010, MNRAS, 401, 1166

Draine, B. T. \& Bertoldi, F. 1996, ApJ, 468, 269

Fumagalli, M., Krumholz, M. R., \& Hunt, L. K. 2010, ApJ, 722, 919

Glover, S. C. O., \& Clark, P. C. 2012a, MNRAS, 421, 9

-. 2012b, MNRAS, in press, arXiv:1203.4251

Glover, S. C. O. \& Mac Low, M.-M. 2007, ApJ, 659, 1317

Gnedin, N. Y. \& Kravtsov, A. V. 2010, ApJ, 714, 287

Gnedin, N. Y., Tassis, K., \& Kravtsov, A. V. 2009, ApJ, 697, 55

Hopkins, A. M. \& Beacom, J. F. 2006, ApJ, 651, 142

Kawamura, A., Mizuno, Y., Minamidani, T., et al. 2009, ApJS, 184, 1

Kennicutt, Jr., R. C. 1998, ARA 6 A, 36, 189

Kennicutt, Jr., R. C., Calzetti, D., Walter, F., et al. 2007, ApJ, 671, 333

Krumholz, M. R. 2012, ApJ, in press, arXiv:1208.1504

Krumholz, M. R. \& Dekel, A. 2012, ApJ, 753, 16

Krumholz, M. R., Ellison, S. L., Prochaska, J. X., \& Tumlinson, J. 2009a, ApJL, 701, L12

Krumholz, M. R. \& Gnedin, N. Y. 2011, ApJ, 729, 36

Krumholz, M. R., Leroy, A. K., \& McKee, C. F. 2011, ApJ, 731, 25

Krumholz, M. R., McKee, C. F., \& Tumlinson, J. 2008, ApJ, 689, 865

-. 2009b, ApJ, 693, 216

- 2009c, ApJ, 699, 850

Kuhlen, M., Krumholz, M. R., Madau, P., Smith, B. D., \& Wise, J. 2012, ApJ, 749, 36

Lee, M.-Y., Stanimirović, S., Douglas, K. A., et al. 2012, ApJ, 748, 75

Leroy, A. K., Walter, F., Brinks, E., et al. 2008, AJ, 136, 2782

Leroy, A. K., Bolatto, A., Gordon, K., et al. 2011, ApJ, 737, 12

Liszt, H. 2002, $A \mathscr{E} A, 389,393$

Mac Low, M.-M. \& Glover, S. C. O. 2012, ApJ, 746, 135

McKee, C. F. \& Krumholz, M. R. 2010, ApJ, 709, 308

Neistein, E., van den Bosch, F. C., \& Dekel, A. 2006, MNRAS, 372, 933

Neufeld, D. A. \& Spaans, M. 1996, ApJ, 473, 894

Omukai, K., Hosokawa, T., \& Yoshida, N. 2010, ApJ, 722, 1793

Rafelski, M., Wolfe, A. M., \& Chen, H.-W. 2011, ApJ, 736, 48

Robertson, B. E. \& Bullock, J. S. 2008, ApJL, 685, L27

Saintonge, A., Kauffmann, G., Wang, J., et al. 2011, MNRAS, 61

Schaye, J., Dalla Vecchia, C., Booth, C. M., et al. 2010, MNRAS, 402, 1536

Schruba, A., Leroy, A. K., Walter, F., et al. 2011, AJ, 142, 37

Sternberg, A. 1988, ApJ, 332, 400

van Dishoeck, E. F. \& Black, J. H. 1986, ApJS, 62, 109

Wild, V., Hewett, P. C., \& Pettini, M. 2007, MNRAS, 374, 292

Wolfe, A. M. \& Chen, H.-W. 2006, ApJ, 652, 981

Wolfire, M. G., Tielens, A. G. G. M., Hollenbach, D., \& Kaufman, M. J. 2008, ApJ, 680, 384

Wong, T. \& Blitz, L. 2002, ApJ, 569, 157 